\title{
Ondelettes and Phase Cell Cluster Expansions, A Vindication*
}

\author{
Guy Battle ${ }^{1 \dagger}$ and Paul Federbush ${ }^{2}$ \\ 1 Department of Mathematics, Cornell University, Ithaca, NY 14850, USA \\ 2 Department of Mathematics, University of Michigan, Ann Arbor, MI 48109, USA
}

\begin{abstract}
Y. Meyer has recently developed a particularly useful o.n. basis for $L^{2}\left(R^{d}\right)$. Expansions using this basis, i.e. expansions into "ondelettes" or "wavelets," have yielded important new results in soft and hard analysis. The expansion into ondelettes of a boson scalar field naturally leads to phase cell cluster expansions, a formalism already developed by the authors using other related bases. Adoption of ondelettes expansions into the phase cell program gives improvements of some extant results, and excises an early error.

Ondelettes lend more elegance to the phase cell cluster expansion of $\phi_{3}^{4}$, and to us are a vindication of the fundamental nature of this approach. This provides more promise for future developments.
\end{abstract}

\section{Ondelettes}

We consider compatible lattices $\mathscr{L}^{r}$ in $R^{d}$ of edge size $L_{r}=\left(1 / 2^{r}\right), r=0, \pm 1, \pm 2, \ldots$ We now state some of the properties of the o.n. basis $\left\{\psi_{k}\right\}$ developed by Y. Meyer associated to these lattices [5].

1) Each $\psi_{k}$ is associated to some cube, in some $\mathscr{L}^{r}, r=r(k)$. There are the same number of $\psi_{k}$ associated to each cube. Given the set of $\psi_{k}$ associated to any cube, the set of $\psi_{k}$ associated to any other cube are the natural dilation, translation, and multiple of these $\psi_{k}$. More exactly, there are a number of functions $f_{1}, \ldots, f_{\alpha}$ such that the $\psi_{k}$ associated to cube $\gamma$ in $\mathscr{L}^{r}, r=r(y)$, are

$$
L_{r(y)}^{-d / 2} f_{i}\left(\frac{x-x^{(\eta)}}{L_{r(\gamma)}}\right), \quad i=1, \ldots, \alpha,
$$

where $x^{(y)}$ is the center of $y$.

\footnotetext{
${ }^{\dagger}$ On leave from the Department of Mathematics, Texas A \& M University, College Station, TX 77843, USA

* This work was supported in part by the National Science Foundation under Grants No. PHY 8502074 and
} 
2) $\psi_{k}$ is $C^{\infty}$, its Fourier transform is $C^{\infty}$ and of compact support.

3) $\int \psi_{k} x^{\alpha}=0$, all $\alpha$, i.e. all the moments of the $\psi_{k}$ are zero.

4) $\left|\left(x-x^{(\gamma)}\right)^{\beta} D^{\alpha} \psi_{k}(x)\right| \leqq c_{\alpha, \beta} L_{r}^{|\beta|-|\alpha|-d / 2}$ for compatible $r, \gamma, k$. That is, the $\psi_{k}$ fall of faster than any power, along with their derivatives, with distance from their associated cube (in the appropriate length scale).

\section{Modified Ondelettes}

The basis of Y. Meyer above may be modified to yield a basis $\left\{\psi_{k}^{\prime}\right\}$ with the $\psi_{k}^{\prime}$ associated only to cubes in $\mathscr{L}^{r}, r \geqq 0$. (We have a maximum cube size.) We are indebted to I. Daubechies for this modification [3]. The modified basis satisfies the following properties:

1) $)^{\prime}$ To a cube in $\mathscr{L}^{r}, r>0$, the associated functions are the same as in Sect. 1.

2) To each cube in $\mathscr{L}^{0}$ the associated $\psi_{k}^{\prime}$ include the set of associated $\psi_{k}$, and a finite additional number of functions.

3) The additional functions associated to cubes in $\mathscr{L}^{0}$ need not have zero moments, but they satisfy 2) and 4) above. The additional functions associated to any cube in $\mathscr{L}^{0}$ are the natural translates of the additional functions associated to any other cube in $\mathscr{L}^{0}$.

\section{Sharply Localized Bases}

In $[1,2,4$, and 6$]$ one works with bases that have many of the properties of ondelettes; the functions have not all moments zero but some fixed finite number, they are sharply localized (each function vanishing outside the cube to which it is associated). See Sect. 4 of [4] and Sect. 2 of [1]. The functions are not smooth, and this led to a technical error in [1]. These bases will, however, find applications, for example in lattice situations as in [4] where smoothness is not very meaningful; or anywhere where sharp localization is more important than smoothness.

\section{Exponentially Localized Bases}

These is an o.n. basis satisfying 1) of Sect. 1, made up of $C^{\infty}$ functions, and having exponential fall off, instead of the faster than any power fall off of 4 ) of Sect. 1. This basis may be developed having any fixed finite number of moments equal to zero. It also may be modified to have a maximal cube size.

This basis was developed by P. -G. Lemarié, and independently by G. Battle. For studying fall off properties of correlation functions in field theory, these bases may be better to work with than the modified ondelettes.

\section{Phase Cell Cluster Expansions}

The phase cell procedure for a boson scalar field theory begins with an expansion of the field

$$
\phi(x)=\sum_{k} \alpha_{k} u_{k}(x)
$$


The $\alpha_{k}$ are the variables describing the field. The $u_{k}(x)$ have a hierarchical nature (i.e. they have property 1) of Sect. 1, or its generalization to a situation with a maximum cube size). Each $u_{k}(x)$ represents an excitation, approximately localized in some region, and with characteristic momenta at the length scale of the localization. The phase cell cluster expansion is an expression for suitable expectation values as a sum of contributions due to finite subsets of the variables. The cluster expansion is developed by iterated decoupling of larger and larger subsets of the variables, just as the Mayer series in classical statistical mechanics is developed by the iterated decoupling of larger and larger subsets of particles.

The first model so treated was an infrared lattice model in [4]. $P(\phi)_{2}$ models were treated in [1] (but with a lamentable error; (3.7) and (3.8) therein are incorrect). The hierarchical $\phi_{3}^{4}$ model was treated in [2]. The full $\phi_{3}^{4}$ model was treated by C. Williamson in [6]. The papers [1,2, and 4] have one elegant feature lacking in [6], the coupling between different variables $\alpha_{k}$ comes entirely from the interaction terms, the free action is diagonal in the variables.

With the incorporation of ondelettes the picture above is aesthetically improved. Working with $u_{k}(x)=\left(1 / \sqrt{-\Delta+M^{2}}\right) \psi_{k}^{\prime}(x)\left(\psi_{k}^{\prime}\right.$ the modified ondelettes or a basis from Sect. 4), instead of the similar expression in (1.3) of [1] in terms of a sharply localized basis, the $P(\phi)_{2}$ paper [1] becomes correct, with no other changes. (Merely replace Sect. 2 therein by a description of the modified ondelettes.) Working with this same choice for $u_{k}(x)$ the hierarchical $\phi_{3}^{4}$ paper [2] becomes a treatment of the actual $\phi_{3}^{4}$ model. It is a treatment of the $\phi_{3}^{4}$ model with the attractive feature mentioned above.

The error in [1], really of a nonbasic nature, and not effecting the correctness of the further papers, had the unfortunate result of distracting favor from the phase cell approach. Further, the initial treatment of $\phi_{3}^{4},[6]$, lacked some of the elegance of the work in [1 and 2]. (It does, though, contain some interesting new twists, possibly of use in later developments.) The entrance of ondelettes provides a happy ending a promising beginning of further progress.

\section{References}

1. Battle, G., Federbush, P.: Ann. Phys. 142, 95-139 (1982)

2. Battle, G., Federbush, P.: Commun. Math. Phys. 88, 263-293 (1983)

3. Daubechies, I.: Private communication

4. Federbush, P.: Commun. Math. Phys. 81, 327--340 and 341-360 (1981)

5. Meyer, Y.: La transformation en ondelettes (to be published)

6. Williamson, C.: A phase cell cluster expansion for $\phi_{3}^{4}$, Missouri. Preprint

Communicated by A. Jaffe

Received August 12, 1986; in revised form September 29, 1986 